\title{
On the K-Fibonacci Hankel and the 4 X 4 Skew Symmetric K- Fibonacci Matrices.
}

\author{
Sergio Falcon \\ University of Las Palmas de Gran Canaria, Department of Mathematics Las Palmas de Gran Canaria, Spain.
}

\begin{abstract}
In this paper we define the k-Fibonacci Hankel matrices and then we study the different norms of these matrices. Next we find the relation between the Euclidean norm, the column norm and the spectral norm of these special matrices. Finally, we study the $4 x 4$ skew symmetric $k$-Fibonacci matrices and find an interesting formula for a sum of the k-Fibonacci numbers.
\end{abstract}

Keywords: k-Fibonacci and k-Lucas numbers; Skew symmetric and Hankel matrices; Matrix Norms.

\section{Introduction}

One of the more studied sequences is the Fibonacci sequence [8,9], and it has been generalized in many ways [7]. Here, we use the following one-parameter generalization of the Fibonacci sequence [3, 4].

\subsection{Definition of the k-Fibonacci numbers.}

For any integer $k \geq 1$, the k-Fibonacci sequence, say $\left\{F_{k, n}\right\}_{n \in N}$ isdefined recurrently by: $F_{k, 0}=0, F_{k, 1}=1$ and $F_{k, n+1}=k F_{k, n}+F_{k, n-1}$

Note that for $\mathrm{k}=\$$ the classical Fibonacci sequence is obtained and for $\mathrm{k}=2$ it is the Pell sequence.

Characteristic equation of the definition is $r^{2}=k r+1$ whose solutions are $\sigma_{1}=\frac{k+\sqrt{k^{2}+4}}{2}$ and $\sigma_{2}=\frac{k-\sqrt{k^{2}+4}}{2}$. It is easy to prove that these solutions verify the following relations: $\sigma_{1} \cdot \sigma_{2}=-1, \sigma_{1}+\sigma_{2}=k, \sigma_{1}-\sigma_{2}=\sqrt{r^{2}+4}, \sigma^{2}=k \sigma+1, \sigma_{1}>0, \sigma_{2}<0$.

For the properties of the k-Fibonacci numbers, see [3, 4].

In particular, the Binet Identity for the k-Fibonacci numbers is $F_{k, n}=\frac{\sigma_{1}^{n}-\sigma_{2}^{n}}{\sigma_{1}-\sigma_{2}}$ and the Catalan Identity is

$$
F_{k, n-r} F_{k, n+r}-F_{k, n}^{2}=(-1)^{n+1-r} F_{k, r}^{2}
$$

From the Catalan Identity, we find the Simson Identity:

$$
F_{k, n-1} F_{k, n+1}=F_{k, n}^{2}+(-1)^{n}
$$

Moreover, $\sum_{j=0}^{n} F_{k, j}=\frac{1}{k}\left(F_{k, n+1}+F_{k, n}-1\right)$ and the sum of the odd k-Fibonacci numbers is

$\sum_{j=0}^{n} F_{k, 2 j+1}=\frac{1}{k} F_{k, 2 n+2}$

Finally, we define the k-Fibonacci numbers of negative index as $F_{k,-n}=(-1)^{n+1} F_{k, n}$

If $F_{k, 0}=2$ instead 0 , we obtain the k-Lucas numbers $L_{k, n}$. Among its properties, highlight the following: $L_{k, n}=\sigma_{1}^{n}+\sigma_{2}^{n}, L_{k, n}=F_{k, n-1}+F_{k, n+1}, L_{k,-n}=(-1)^{n} L_{k, n}$. See [2] for the properties of these numbers.

1.2. Some formulas for the k-Fibonacci numbers.

In [1], the following theorem is proved: For $h \geq 0$, the sum of the products of two k-Fibonacci numbers is

$$
S_{m, r}=\sum_{j=1}^{m} F_{k, j} F_{k, j+r}=\frac{1}{k}\left(F_{k, m+1} F_{k, m+r}-\frac{(-1)^{m}+1}{2} F_{k, r}\right)
$$


From this formula we will find an expression for the number $F_{k, n}$ :

$$
\begin{aligned}
S_{m-1}, r & =\sum_{j=1}^{m-1} F_{k, j} F_{k, j+r}=\sum_{j=1}^{m} F_{k, j} F_{k, j+r}-F_{k, m} F_{k, m+r}=\frac{1}{k}\left(F_{k, m+1} F_{k, m+r}-\frac{(-1)^{m}+1}{2} F_{k, r}\right)-F_{k, m} F_{k, m+r} \\
& =\frac{1}{k}\left(\left(F_{k, m+1}-k F_{k, m}\right) F_{k, m+r}-\frac{(-1)^{m}+1}{2} F_{k, r}\right)=\frac{1}{k}\left(F_{k, m-1} F_{k, m+r}-\frac{(-1)^{m}+1}{2} F_{k, m+r}\right)
\end{aligned}
$$

from the formula (1.3) and directly, it is $S_{m-1, r}=\frac{1}{k}\left(F_{k, m} F_{k, m+r-1}-\frac{(-1)^{m-1}+1}{2} F_{k, r}\right)$ and equalizing these expressions it is $F_{k, r}=F_{k, m} F_{k, m+r-1}-F_{k, m-1} F_{k, m+r}$.

Particular cases:

$$
\begin{aligned}
& S_{m, 0}=\sum_{j=1}^{m} F_{k, j}^{2}=\frac{1}{k} F_{k, m} F_{k, m+1} \\
& S_{m-1,1}=\sum_{j=1}^{m-1} F_{k, j} F_{k, j+1}=\frac{1}{k}\left(F_{k, m-1} F_{k, m+1}-\frac{(-1)^{m}+1}{2}\right) \\
& S_{2 m-1,1}=\sum_{j=1}^{2 m-1} F_{k, j} F_{k, j+1}=\frac{1}{k}\left(F_{k, 2 m-1} F_{k, 2 m+1}-1\right)=\frac{1}{k} F_{k, 2 m}^{2}
\end{aligned}
$$

\subsection{Matrix norms.}

The following matrix norms are defined in $[5,10]$.

Let $A=\left(a_{i j}\right)$ be an $m \times n$ matrix.

- The Frobenius or Euclidean norm of the matrix A is defined as $\|A\|_{E}=\left(\sum_{i=1}^{m} \sum_{j=1}^{n}\left|a_{i j}\right|^{2}\right)^{1 / 2}$

- The column norm of $\mathrm{A}$ is defined as $\|A\|_{1}=\max _{1 \leq j \leq n} \sum_{i=1}^{m}\left|a_{i j}\right|$, which is simply the maximum absolute column sum of the matrix.

- The row norm of $\mathrm{A}$ is $\|A\|_{\infty}=\max _{1 \leq j \leq m} \sum_{j=1}^{m}\left|a_{i j}\right|$, which is simply the maximum absolute row sum of the matrix.

- The spectral norm of $\mathrm{A}$ is the largest singular value of $\mathrm{A}$ i.e. the square root of the largest eigenvalue of the positive-semidefinite matrix $A^{*} A$ where $\mathrm{A}^{*}$ denotes the conjugate transpose of $\mathrm{A}$; that is $\|A\|_{2}=\sqrt{\lambda_{\max }\left(A^{*} A\right)}=\sigma_{\text {max }}(A)$

Evidently, if $\mathrm{A}$ is an $\mathrm{x} n$ symmetric matrix, the column norm and the row norm coincide and the spectral norm is the absolutely biggest eigenvalue of A called the spectral radius.

\subsection{Principal minors of a matrix.}

Let A be an $m \times n$ matrix and $h$ an integer with $0<h \leq m$, and $h \leq n$. A $h \times h$ minor of A is the determinant of a $h \times h$ matrix obtained from A by deleting $\mathrm{m}-\mathrm{h}$ rows and $\mathrm{n}-$ hcolumns [11].

If $\mathrm{A}$ is an $m \mathrm{x} n$ matrix, $\mathrm{I}$ is a subset of $\{1,2, \ldots, m\}$ having $h$ elements and $\mathrm{J}$ is a subset of $\{1,2, \ldots, n\}$ with $h$ elements, then we write $[A]_{I, J}$ for the $h \times h$ minor of A that corresponds to the rows with index in I and the columns with index in $\mathrm{J}$.

If $\mathrm{I}=\mathrm{J}$, then $[A]_{I, I}$ is called a principal minor of $\mathrm{A}$ and we will indicate it as $[A]_{h}$.

Characteristic polynomial of an $n \times n$ matrix $\mathrm{A}$ is $P(\lambda)=(-1)^{n}\left(\lambda^{n}-\sum_{h=1}^{n}[A]_{h} \lambda^{n-h}\right)$. Evidently, $[A]_{1}=\operatorname{trace}(A)$ and $[A]_{n}=\operatorname{det}(A)$. 


\section{On The K-Fibonacci Hankel Matrices.}

Let the sequence $\left\{t_{l}\right\}_{l \in N}$ be. The Hankel matrix of this sequence is the $n \times n$ matrix $\left(a_{i j}\right)$ such that $a_{i j}=t_{i+j-1}$. If all $t_{l}$ are the k-Fibonacci numbers, $t_{l}=F_{k, l}$, the k-Fibonacci Hankel matrix is defined as the $n \times n$ matrix $\left(a_{i j}\right)$ such that $a_{i j}=F_{k, i+j-1}$; i.e.

$$
\left(F_{k} H\right)_{n}=\left(\begin{array}{cccccc}
F_{k, 1} & F_{k, 2} & F_{k, 3} & \cdots & F_{k, n-1} & F_{k, n} \\
F_{k, 2} & F_{k, 3} & F_{k, 4} & \cdots & F_{k, n} & F_{k, n+1} \\
F_{k, 3} & F_{k, 4} & F_{k, 5} & \cdots & F_{k, n+1} & F_{k, n+2} \\
\vdots & \vdots & \vdots & \ddots & \vdots & \vdots \\
F_{k, n} & F_{k, n+1} & F_{k, n+2} & \cdots & F_{k, 2 n-2} & F_{k, 2 n-1}
\end{array}\right)
$$

It is obvious that all k-Fibonacci Hankel matrix is symmetric. Moreover, $\left|\left(F_{k} H\right)_{1}\right|=\left|\left(F_{k} H\right)_{2}\right|=1$, and for $n \geq 3$ it is $\left|\left(F_{k} H\right)_{n}\right|=0$ because each row is a linear combination of the two preceding rows.

Moreover, the characteristic polynomial of $\left(F_{k} H\right)_{1}$ is $P_{1}(\lambda)=1-\lambda$ and that of $\left(F_{k} H\right)_{2}$ is $P_{2}(\lambda)=\lambda^{2}-\left(k^{2}+2\right) \lambda+1$.

\subsection{Theorem 1}

For $n \geq 1$, the characteristic polynomial of the k-Fibonacci Hankel matrix $\left(F_{k} H\right)_{n}$ is

$$
P(\lambda)=(-1)^{n} \lambda^{n-2}\left(\lambda^{2}-\frac{F_{k, 2 n}}{k} \lambda+\frac{1}{k^{2}}\left(F_{k, n}^{2}+\frac{(-1)^{n}-1}{2}\right)\right)
$$

Proof. For $\mathrm{n}=1,2$, the preceding equations coincide with this last equation for $\mathrm{n}=1$ and $\mathrm{n}=2$, respectively.

For $n \geq 3$, each row from the third is a linear combination of the two preceding rows. Then all principal minor $\left[F_{k} H\right]_{h}=0$ for $h \geq 3$ and consequently, the only non-null coefficients of the characteristic polynomial (other than $\lambda^{n}$ is 1) are those of $\lambda^{n-1}$ and $\lambda^{n-2}$.

The coefficient of $\lambda^{n-1}$ is the sum of the principal minors of order 1 :

$$
\sum_{j=1}^{n}\left(\left|F_{k} H\right|_{1}\right)_{j}=\operatorname{trace}\left(F_{k} H\right)=\sum_{j=1}^{n} F_{k, 2 j-1}=\frac{1}{k} F_{k, 2 n} \text {. }
$$

The coefficient of $\lambda^{n-1}$ is the sum of the principal minors of order 2 . To find this coefficient, we will apply the Catalan Identity (1.1) to the principal minors of order two.

First row: The sum of the principal minors whose first entry is $F_{k, 1}$, after applying the formula (1.3) is

$$
S_{1}=\sum_{j=2}^{n}\left|\begin{array}{cc}
F_{k, 1} & F_{k, j} \\
F_{k, j} & F_{k, 2 j-1}
\end{array}\right|=\sum_{j=2}^{n}\left(F_{k, 1} F_{k, 2 j-1}-F_{k, j}^{2}\right)=\sum_{j=1}^{n-1} F_{k, j}^{2}=\frac{1}{k} F_{k, n-1} F_{k, n}
$$

Second row: The sum of the principal minors whose first entry is $F_{k, 3}$, is $S_{2}=\sum_{j=4}^{n}\left|\begin{array}{cc}F_{k, 1} & F_{k, j} \\ F_{k, j} & F_{k, 2 j-1}\end{array}\right|=\sum_{j=4}^{n}\left(F_{k, 3} F_{k, 2 j-1}-F_{k, j}^{2}\right)=\sum_{j=1}^{n-2} F_{k, j}^{2}=\frac{1}{k} F_{k, n-2} F_{k, n-1}$

The sum of the principal minors whose first entry is $F_{k, 5}$, is $S_{3}=\frac{1}{k} F_{k, n-3} F_{k, n-2}$, etc.

Finally, and taking into account the formula (1.3), the sum of all principal minors of order 2 of the k-Fibonacci Hankel matrix is $\frac{1}{k} \sum_{j=1}^{n-2} F_{k, n-j} F_{k, n-j+1}=\frac{1}{k}\left(\frac{1}{k}\left(F_{k, n-1}^{2}+\frac{(-1)^{n}-1}{2}\right)\right)$, and this is the coefficient of $\lambda^{n-1}$. 
1.6. Eigenvalues of $\left(F_{k} H\right)_{n}$

Evidently, $\left(F_{k} H\right)_{n}$ has $\mathrm{n}-2$ null eigenvalues and the non-singular eigenvalues are $\lambda_{1,2}=\frac{1}{2 k}\left(F_{k, 2 n} \pm \sqrt{F_{2 n}^{2}-4\left(F_{k, n}^{2}+\frac{(-1)^{n}-1}{2}\right)}\right)$. Of course, taking into account this matrix is symmetric, these two non-singular eigenvalues are real.

And the spectral norm of the k-Fibonacci Hankel matrix $\left(F_{k} H\right)_{n}$ is $\left\|\left(F_{k} H\right)_{n}\right\|_{2}=\lambda_{1}=\frac{1}{2 k}\left(F_{k, 2 n}+\sqrt{F_{2 n}^{2}-4\left(F_{k, n}^{2}+\frac{(-1)^{n}-1}{2}\right)}\right)$

\subsection{Theorem 2}

The Euclidean norm of the k-Fibonacci Hankel matrix is $\left\|\left(F_{k} H\right)_{n}\right\|_{E}=\frac{1}{k} \sqrt{F_{k, 2 n}^{2}-2 F_{k, n}^{2}-(-1)^{n}+1}$

Proof. Summing the squares of the entries by rows, and taking into account Formula (1.2) and later (1.4) and (1.3), the Euclidean norm of the k-Fibonacci Hankel matrix is:

$$
\begin{aligned}
\left(\left\|\left(F_{k} H\right)_{n}\right\|_{E}\right)^{2} & =\sum_{j=1}^{n} F_{k, j}^{2}+\sum_{j=2}^{n+1} F_{k, j}^{2}+\sum_{j=3}^{n+2} F_{k, j}^{2}+\cdots+\sum_{j=n}^{2 n-1} F_{k, j}^{2} \\
& =\left(\sum_{j=1}^{n} F_{k, j}^{2}+\sum_{j=1}^{n+1} F_{k, j}^{2}+\sum_{j=1}^{n+2} F_{k, j}^{2}+\cdots+\sum_{j=1}^{2 n-1} F_{k, j}^{2}\right)-\left(\sum_{j=1}^{n-1} \sum_{i=1}^{j} F_{k, i}^{2}\right) \\
& =\frac{1}{k}\left(F_{k, n} F_{k, n+1}+F_{k, n+1} F_{k, n+2}+F_{k, n+2} F_{k, n+3}+\cdots+F_{k, 2 n-1} F_{k, 2 n}\right)-\frac{1}{k} \sum_{j=1}^{n-1} F_{k, j} F_{k, j+1} \\
& =\frac{1}{k}\left(\sum_{j=1}^{2 n-1} F_{k, j} F_{k, j+1}-2 \sum_{j=1}^{n-1} F_{k, j} F_{k, j+1}\right)=\frac{1}{k}\left(\frac{1}{k} F_{k, 2 n}^{2}-\frac{2}{k}\left(F_{k, n-1} F_{k, n+1}-\frac{(-1)^{n}+1}{2}\right)\right) \\
& =\frac{1}{k^{2}}\left(F_{k, 2 n}^{2}-2 F_{k, n-1} F_{k, n+1}+(-1)^{n}+1\right)=\frac{1}{k^{2}}\left(F_{k, 2 n}^{2}-2 F_{k, n}^{2}-(-1)^{n}+1\right)
\end{aligned}
$$

after applying the Simson Identity and the proof is complete.

If $\mathrm{n}=1$, then $\left\|\left(F_{k} H\right)_{1}\right\|_{E}=\frac{1}{k} \sqrt{F_{k, 2}^{2}-2 F_{k, 1}^{2}+2}=1$.

Taking into account the expressions of the eigenvalues, it is easy to prove $\left\|\left(F_{k} H\right)_{n}\right\|_{E}=\sqrt{\lambda_{1}^{2}+\lambda_{2}^{2}}$ and, consequently, $\left\|\left(F_{k} H\right)_{n}\right\|_{2}<\left\|\left(F_{k} H\right)_{n}\right\|_{E}$.

\subsection{Theorem 3}

The column norm (or the row norm) of the k-Fibonacci Hankel matrix is $\left\|\left(F_{k} H\right)_{n}\right\|_{1}=\left\|\left(F_{k} H\right)_{n}\right\|_{\infty}=\frac{1}{k}\left(F_{k, 2 n-1}+F_{k, 2 n}-F_{k, n-1}-F_{k, n}\right)$

Proof. From the definition of k-Fibonacci numbers, $k \geq 1$, and consequently, the sequence $F_{k}=\left\{F_{k, n}\right\}_{n \in N}$ is positive and increasing. So, and taking into account the sum formula, $\left\|\left(F_{k} H\right)_{n}\right\|_{1}=\sum_{j=n}^{2 n-1} F_{k, j}=\sum_{j=1}^{2 n-1} F_{k, j}-\sum_{j=1}^{n-1} F_{k, j}=\frac{1}{k}\left(F_{k, 2 n-1}+F_{k, 2 n}-F_{k, n-1}-F_{k, n}\right)$.

In particular, for the classical Fibonacci numbers $\left\|(F H)_{n}\right\|_{1}=F_{2 n+1}-F_{n+1}$.

And if $\mathrm{n}=1,\left\|\left(F_{k} H\right)_{1}\right\|_{1}=\frac{1}{k}\left(F_{k, 1}+F_{k, 2}-F_{k, 0}-F_{k, 1}\right)=1$. 


\subsection{Theorem 4}

For $\mathrm{n}>1,\left\|\left(F_{k} H\right)_{n}\right\|_{E}<\left\|\left(F_{k} H\right)_{n}\right\|_{1}$

Proof. We must prove

$$
\begin{aligned}
& F_{k, 2 n}^{2}-2 F_{k, n}^{2}-(-1)^{n}+1<k\left(F_{k, 2 n-1}+F_{k, 2 n}-F_{k, n}-F_{k, n-1}\right)^{2} \\
& =k\left(F_{k, 2 n-1}^{2}+F_{k, 2 n}^{2}+F_{k, n-1}^{2}+F_{k, n}^{2}\right)+2 k\left(F_{k, 2 n-1} F_{k, 2 n}+F_{k, n-1} F_{k, n}\right)-2 k\left(F_{k, 2 n-1} F_{k, 2 n}\right)\left(F_{, n-1} F_{k, n}\right)
\end{aligned}
$$

But taking into account $F_{k, 2 n}^{2} \leq k F_{k, 2 n}^{2}$ and $-2 F_{k, n}^{2}<k F_{k, n}^{2}$, the proof is complete.

In short: for $\mathrm{n}>1$, the relationship $\left\|\left(F_{k} H\right)_{n}\right\|_{2}<\left\|\left(F_{k} H\right)_{n}\right\|_{E}<\left\|\left(F_{k} H\right)_{n}\right\|_{1}$ is verified.

\section{On The 4 X 4 Skew Symmetric K-Fibonacci Matrices.}

A $n \times n$ matrix $\left(a_{i, j}\right)$ is skew symmetric if for all $i, j$ it is $a_{i, j}=-a_{j, i}$ [12]. Consequently, all the elements of the principal diagonal are null and then $\operatorname{tr}(\mathrm{A})=0$. Moreover, if $n$ is odd, $\operatorname{det}(\mathrm{A})=0$.

Let us consider the skew symmetric matrix $M=\left(\begin{array}{cccc}0 & a & -b & c \\ -a & 0 & d & e \\ b & -d & 0 & f \\ -c & -e & -f & 0\end{array}\right)$

Then, $\operatorname{det}(M)=(a f+b e+c d)^{2}$, its Euclydean norm is $\|M\|_{E}=\sqrt{2\left(a^{2}+b^{2}+c^{2}+d^{2}+e^{2}+f^{2}\right)}$, its characteristic equation is $\lambda^{4}+\frac{1}{2} \sqrt{\|M\|_{E}} \lambda^{2}+\operatorname{det}(M)=0$, and consequently, any eigenvalue is real.

\subsection{On the $4 \times 4$ skew symmetric k-Fibonacci matrices.}

Let us suppose the entries in the previous matrix are $a=F_{k, r+1}, b=F_{k, r+2}, \ldots, f=F_{k, r+6}$ Then, the $4 \times 4$

skew symmetric k-Fibonacci matrix has the form $(F M)=\left(\begin{array}{cccc}0 & F_{k, r+1} & -F_{k, r+2} & F_{k, r+3} \\ -F_{k, r+1} & 0 & F_{k, r+4} & F_{k, r+5} \\ F_{k, r+2} & -F_{k, r+4} & 0 & F_{k, r+6} \\ -F_{k, r+3} & -F_{k, r+5} & -F_{k, r+6} & 0\end{array}\right)$

According to the above formula it is $\operatorname{det}(F M)=\left(F_{k, r+1} F_{k, r+6}+F_{k, r+2} F_{k, r+5}+F_{k, r+3} F_{k, r+4}\right)^{2}$ and it is easy to prove $\|(F M)\|_{E}^{2}=\frac{2}{k\left(k^{2}+4\right)}\left(L_{k, 2 r+13}-L_{k, 2 r+1}\right)$

1.11. Determinant of the $\$ 4 \mid t i m e s \$$ skew symmetric k-Fibonacci matrix.

We expand the formula of the determinant in order to obtain a more reduced formula. We will use the Binnet

$$
\begin{aligned}
& \left(k^{2}+4\right)\left(F_{k, r+1} F_{k, r+6}+F_{k, r+2} F_{k, r+5}+F_{k, r+3} F_{k, r+4}\right) \\
= & \left(\sigma_{1}^{r+1}-\sigma_{2}^{r+1}\right)\left(\sigma_{1}^{r+6}-\sigma_{2}^{r+6}\right)+\left(\sigma_{1}^{r+2}-\sigma_{2}^{r+2}\right)\left(\sigma_{1}^{r+5}-\sigma_{2}^{r+5}\right)+\left(\sigma_{1}^{r+3}-\sigma_{3}^{r+1}\right)\left(\sigma_{1}^{r+4}-\sigma_{2}^{r+4}\right) \\
\text { Identity. }= & \sigma_{1}^{2 r+7}+\sigma_{2}^{2 r+7}-(-1)^{r+1}\left(\sigma_{1}^{5}+\sigma_{2}^{5}\right)+\sigma_{1}^{2 r+7}+\sigma_{2}^{2 r+7}-(-1)^{r}\left(\sigma_{1}^{3}+\sigma_{2}^{3}\right)+\sigma_{1}^{2 r+7} \\
& +\sigma_{2}^{2 r+7}-(-1)^{r+1}\left(\sigma_{1}+\sigma_{2}\right) \\
= & 3 L_{k, 2 r+7}+(-1)^{r}\left(L_{k, 5}-L_{k, 3}+L_{k, 1}\right)=3 L_{k, 2 r+7}+(-1)^{r} F_{k, 6}
\end{aligned}
$$

from where

$$
\begin{aligned}
& F_{k, r+1} F_{k, r+6}+F_{k, r+2} F_{k, r+5}+F_{k, r+3} F_{k, r+4}=\frac{1}{k^{2}+4}\left(3 L_{k, 2 r+7}+(-1)^{r} F_{k, 6}\right) \\
& \rightarrow \operatorname{det}(F M)=\left(\frac{3 L_{k, 2 r+7}+(-1)^{r} F_{k, 6}}{k^{2}+4}\right)^{2}
\end{aligned}
$$


And finally, $\quad F_{k, r+1} F_{k, r+6}+F_{k, r+2} F_{k, r+5}+F_{k, r+3} F_{k, r+4}=\frac{1}{k^{2}+4}\left(3 L_{k, 2 r+7}+(-1)^{r} F_{k, 6}\right)$

For instance: for $\mathrm{k}=1 \mathrm{nd} \mathrm{r}=0$, it is $F_{1} F_{6}+F_{2} F_{5}+F_{3} F_{4}=\frac{1}{5}(3 \cdot 29+8)=19$

\subsection{Extension of this last formula.}

Now we will try to find an extension of this last formula for a general case with the condition $n$ is even. To prove it, we need the following formulas [2]:

$$
\begin{aligned}
& \sigma_{1} \sigma_{2}=-1 \rightarrow\left(\frac{\sigma_{1}}{\sigma_{2}}\right)^{j}=(-1)^{j} \sigma_{2}^{-2 j} \text { and }\left(\frac{\sigma_{2}}{\sigma_{1}}\right)^{j}=(-1)^{j} \sigma_{1}^{-2 j} \\
& \sum_{j=0}^{n}(-1)^{j} L_{k, a j+r}=\frac{(-1)^{n} L_{k, a(n+1)+r}+(-1)^{a+n} L_{k, a n+r}+(-1)^{r} L_{k, a-r}+L_{k, r}}{L_{k, a}+(-1)^{a}+1}
\end{aligned}
$$

If $\mathrm{a}=2$ and $\mathrm{r}=-2 \mathrm{n}-1$, then

- $\quad(-1)^{n} L_{k, a(n+1)+r}=(-1)^{n} L_{k, 2 n+2-2 n-1}=(-1)^{n} L_{k, 1}=(-1)^{n} k$

- $\quad(-1)^{a+n} L_{k, a n+r}=(-1)^{n} L_{k, 2 n-2 n-1}=(-1)^{n} L_{k,-1}=-(-1)^{n} k$

- $\quad(-1)^{r} L_{k, a-r}=-L_{k, 2+2 n+1}=-L_{k, 2 n+3}$

- $\quad L_{k, r}=L_{k,-2 n-1}=-L_{k, 2 n+1}$

- $\quad L_{k, a}+(-1)^{a}-1=L_{k, 2}+1+1=k^{2}+4$

and the formula (3.3) takes the form

$$
\sum_{j=0}^{n}(-1)^{j} L_{k, 2 j-2 n-1}=\frac{-1}{k^{2}+4}\left(L_{k, 2 n+3}+L_{k, 2 n+1}\right)
$$

\subsubsection{Lemma}

For all $n$, it is

$$
\left(k^{2}+3\right) L_{k, 2 n+1}-L_{k, 2 n+3}=\left(k^{2}+4\right) F_{k, 2 n}
$$

Proof. The Left Hand Side of this formula is

$$
\begin{aligned}
L H S & =\left(\left(\sigma_{1}-\sigma_{2}\right)^{2}-1\right)\left(\sigma_{1}^{2 n+1}+\sigma_{2}^{2 n+1}\right)-\left(\sigma_{1}^{2 n+3}+\sigma_{2}^{2 n+3}\right) \\
& =\left(\sigma_{1}^{2}+\sigma_{2}^{2}+1\right)\left(\sigma_{1}^{2 n+1}+\sigma_{2}^{2 n+1}\right)-\sigma_{1}^{2 n+3}-\sigma_{2}^{2 n+3} \\
& =\sigma_{1}^{2 n+3}+\left(\sigma_{1} \sigma_{2}\right)^{2} \sigma_{1}^{2 n-1}+\sigma_{2}^{2 n+3}+\left(\sigma_{2} \sigma_{1}\right)^{2} \sigma_{2}^{2 n-1}+\sigma_{1}^{2 n+1}+\sigma_{2}^{2 n+1}-\sigma_{1}^{2 n+3}-\sigma_{2}^{2 n+3} \\
& =\sigma_{1}^{2 n-1}+\sigma_{2}^{2 n-1}+\sigma_{1}^{2 n+1}+\sigma_{1}^{2 n+1}
\end{aligned}
$$

And the Right Hand Side is

$$
\begin{aligned}
R H S & =\left(\sigma_{1}-\sigma_{2}\right)^{2} \frac{\sigma_{1}^{2 n}-\sigma_{2}^{2 n}}{\sigma_{1}-\sigma_{2}}=\left(\sigma_{1}-\sigma_{2}\right)\left(\sigma_{1}^{2 n}-\sigma_{2}^{2 n}\right) \\
& =\sigma_{1}^{2 n+1}-\left(\sigma_{1} \sigma_{2}\right) \sigma_{2}^{2 n-1}-\left(\sigma_{2} \sigma_{1}\right) \sigma_{1}^{2 n-1}+\sigma_{2}^{2 n+1}=\sigma_{1}^{2 n-1}+\sigma_{2}^{2 n-1}+\sigma_{1}^{2 n+1}+\sigma_{2}^{2 n+1}=L H S
\end{aligned}
$$

Then we will prove the general formula

$$
\sum_{j=1}^{n} F_{k, r+j} F_{k, r+2 n+1-j}=\frac{1}{k^{2}+4}\left(n L_{k, 2 r+2 n+1}+(-1)^{r} F_{k, 2 n}\right)
$$

Proof. Taking into account the Binet Identity and the formulas (3.2), (3.4), and (3.5): 


$$
\begin{aligned}
\sum_{j=1}^{n} F_{k, r+j} F_{k, r+2 n+1-j} & =\frac{1}{k^{2}+4} \sum_{j=1}^{n}\left(\sigma_{1}^{r+j}-\sigma_{2}^{r+j}\right)\left(\sigma_{1}^{r+2 n+1-j}-\sigma_{2}^{r+2 n+1-j}\right) \\
& =\frac{1}{k^{2}+4} \sum_{j=1}^{n}\left(\sigma_{1}^{2 r+2 n+1}+\sigma_{2}^{2 r+2 n+1}-(-1)^{r} \sigma_{1}^{2 n+1}\left(\frac{\sigma_{2}}{\sigma_{1}}\right)^{j}-(-1)^{r} \sigma_{2}^{2 n+1}\left(\frac{\sigma_{1}}{\sigma_{2}}\right)^{j}\right) \\
& =\frac{1}{k^{2}+4} \sum_{j=1}^{n}\left(L_{k, 2 r+2 n+1}-(-1)^{r}(-1)^{j}\left(\sigma_{1}^{2 n-2 j+1}+\sigma_{2}^{2 n-2 j+1}\right)\right) \\
& =\frac{1}{k^{2}+4} \sum_{j=1}^{n}\left(L_{k, 2 r+2 n+1}-(-1)^{r}(-1)^{j} L_{k, 2 n-2 j+1}\right) \\
& =\frac{1}{k^{2}+4} \sum_{j=1}^{n}\left(L_{k, 2 r+2 n+1}+(-1)^{r}(-1)^{j} L_{k, 2 j-2 n-1}\right) \\
& =\frac{1}{k^{2}+4} \sum_{j=1}^{n}\left(L_{k, 2 r+2 n+1}+(-1)^{r}\left(\sum_{j=0}^{n}(-1)^{j} L_{k, 2 j-2 n-1}-L_{k,-2 n-1}\right)\right) \\
& =\frac{1}{k^{2}+4}\left(n L_{k, 2 r+2 n+1}+(-1)^{r}\left(\frac{1}{k^{2}+4}\left(-L_{k, 2 n+1}-L_{k, 2 n+3}\right)+L_{k, 2 n+1}\right)\right) \\
& =\frac{1}{k^{2}+4}\left(n L_{k, 2 r+2 n+1}+(-1)^{r} \frac{1}{k^{2}+4}\left(\left(k^{2}+3\right) L_{k, 2 n+1}-L_{k, 2 n+3}\right)\right) \\
& =\frac{1}{k^{2}+4}\left(n L_{k, 2 r+2 n+1}+(-1)^{r} F_{k, 2 n}\right)
\end{aligned}
$$

In particular, for $\mathrm{n}=3$, formula (3.1) is obtained.

Finally, similarly to the above formula it can be shown

$$
\sum_{j=1}^{n} F_{k, r+j} F_{k, r+2 n-j}=\frac{1}{k^{2}+4}\left(n L_{k, 2 r+2 n}+(-1)^{r} F_{k, 2 n-1}-(-1)^{r+n}\right)
$$

\section{References}

[1]. S. Falcon, On the sequences of products of two k-Fibonacci numbers, American Review of Mathematics and Statistics, Vol 2(1) (2014), $111-120$.

[2]. $\quad$ S. Falcon, On the $k$-Lucas numbers, Int. J. Contemp. Math. Sciences, Vol. 6(21) (2011), $1039-1050$.

[3]. S. Falcon, A. Plaza, On the Fibonacci $k$-numbers, Chaos, Solit. \&Fract. 32(5) (2007) $1615-24$.

[4]. S. Falcon, A. Plaza, The k-Fibonacci sequence and the Pascal 2-triangle, Chaos, Solit. \&Fract. 33(1) (2007) 38 - 49

[5]. Simon Foucart, http://www.math.drexel.edu/ foucart/TeachingFiles/F12/M504Lect6.pdf

[6]. Serpil Halici, On some inequalities and Hankel Matrices involving Pell, Pell-Lucas numbers, Math. Reports 15(65), 1 (2013), 1 10.

[7]. V.E. Hoggat, Fibonacci and Lucas numbers, Palo Alto, CA: Houghton-Mifflin; 1969.

[8]. A.F. Horadam, A generalized Fibonacci sequence, Math. Mag. 68 (1961) 455 - 9.

[9]. S. Vajda, Fibonaccil\& Lucas Numbers, and the Golden Section, Theory and Applications. Ellis Horwood limited; 1989.

[10]. http://en.wikipedia.org/wiki/Matrix _norm

[11]. http://en.wikipedia.org/wiki/Minor\_(linear\_algebra)

[12]. http://en.wikipedia.org/wiki/Skew--symmetric__matrix 